users (37years. v.41years, $\mathrm{p}=0.2$ ). There were $33 / 38$ clinicians who responded to the online survey. Clinicians felt equally confident at delivering daily PrEP as EBD-PrEP (Likert scores $=4.4 / 5 \mathrm{v}$ 4.2/5, $\mathrm{p}=0.2$ ). However, potential barriers identified to providing EBD-PrEP by clinicians were; assessing which MSM would be suitable for using EBD-PrEP, having access to appropriate information for patients to support their understanding of using EBD PrEP; and clinician knowledge and belief in the efficacy of EBD-PrEP.

Conclusion The uptake by MSM and clinician confidence in discussing EBD-PrEP appears to have increased since the start of the COVID-19 pandemic. Giving MSM greater choice in how PrEP is used will optimise its effect on reducing HIV transmission. More research is needed to support both MSM and clinicians to deliver EBD-PrEP.

\section{P316 COULD NUMBER OF PARTNERS BE A RISK FOR ANTIMICROBIAL RESISTANCE? HIGHER MACROLIDE CONSUMPTION AMONGST A CORE GROUP OF PREP USERS}

${ }^{1,2} \mathrm{C}$ Van Dijck* ${ }^{1} \mathrm{~T}$ Vanbaelen, ${ }^{1,2} \mathrm{~J}_{\text {J Laumen, }}{ }^{1,3} \mathrm{C}$ Kenyon. ${ }^{1}$ Institute of Tropical Medicine Antwerp, Department of Clinical Sciences, HIVISTI Unit, Antwerp, Belgium; ${ }^{2}$ University of Antwerp, Faculty of Medicine, Vaccine and Infectious Disease Institute, Laboratory of Medical Microbiology, Wirrijk, Belgium; ${ }^{3}$ University of Cape Town, Division of Infectious Diseases and HIV Medicine, Cape Town, South Africa

10.1136/sextrans-2021-sti.371

Background A core group of men who have sex with men (MSM) may sustain the high sexually transmitted infection (STI) prevalence among HIV-PrEP users. Regular gonorrhea/ chlamydia screening/treatment in this core group might reduce STI prevalence, but also augments antimicrobial use. Macrolides in particular affect the resistome and may enhance the spreading of antimicrobial resistance. Using data from a singlecenter randomized clinical trial in our clinic we aimed to assess if PrEP-users with multiple sexual partners used more macrolides than others.

Methods Between April 2019 and June 2020, 343 PrEP users participated in a trial that assessed if an antiseptic mouthwash could prevent bacterial STIs (NCT03881007). During 3monthly study visits participants reported their number of casual partners and underwent gonorrhea/chlamydia screening/ treatment at the oropharynx, anorectum and urethra. Macrolide use, expressed as defined daily doses per 1000 individuals per day (DID) was compared between individuals with maximum ten versus more partners per three months.

Results Participants (median age 40.0, IQR 32.0-48.0) contributed for 203.0 person-years. They had a median of 10.0 (IQR 5.6-20.0) partners per three months. Bacterial STI incidence (gonorrhea + chlamydia + syphilis) was 126.6 per 100 person-years. Overall macrolide use was 8.48 DID. Macrolide consumption correlated weakly with number of partners (spearman's rho $0.18, \mathrm{p}<0.001)$. Half $(48.5 \%)$ of the participants had more than ten partners per three months and accounted for $60.3 \%$ of bacterial STIs. At 10.39 DID their macrolide consumption was 1.62 times $(95 \%$ CI 1.38 - 1.91) that of participants with less partners (6.42 DID) and three times $(2.97$, 95\% CI 2.69-3.27) that of the 2019 general Belgian population (3.50 DID).

Conclusion PrEP users that undergo 3-monthly gonorrhea/chlamydia screening use a multitude of the macrolides used by the general population. Especially those with over ten 3- montlhy partners may be at risk to carry and spread antimicrobial resistance.

\section{P317 USING THE HEALTHCARE-OP TOOL TO EVALUATE THE OVERALL COST OF INTRODUCING AN ONLINE SERVICE FOR STI AND HIV SELF-SAMPLING}

${ }^{1} S$ Sundar* ${ }^{*}$ 'S Mohiuddin, ${ }^{2} \mathrm{R}$ Gardiner, ${ }^{3} \mathrm{P}$ Muir, ${ }^{2} \mathrm{H}$ Wheeler, ${ }^{3} \mathrm{~S}$ Steer, ${ }^{3} \mathrm{R}$ Hopes, ${ }^{2} \mathrm{~A}$ Liebow, ${ }^{2} \mathrm{~K}$ Liva-Pye, ${ }^{2} \mathrm{M}$ Griffin, ${ }^{1} \mathrm{P}$ Horner. ${ }^{1}$ Population Health Sciences, University of Bristol, Bristol, UK; ${ }^{2}$ Unity Sexual Health Clinic, Bristol, UK; ${ }^{3}$ South West Regional Public Health Laboratory, Public Health England, Bristol, UK

\subsection{6/sextrans-2021-sti.372}

Background Online services for STI and HIV self-sampling are increasingly being adopted by sexual health services (SHSs). Exploring the impact on overall cost of introducing a new service can be challenging. We developed a simple costing tool (Healthcare-OP) for use by clinicians and managers to support business case preparation for introducing a new service. Healthcare-OP was used to examine the impact on overall cost for the introduction of an online STI and HIV self-sampling service for asymptomatic clients attending our SHS

Methods Client care-pathways were categorised into 16 types based on clinic workflow and estimated duration of consultation. Each pathway was broken down into discrete 'processes,' which were costed based on healthcare professional time and costs of tests/consumables. The probability of clients entering each care-pathway and transitioning between 'processes' in Healthcare-OP was estimated using 12 months electronic patient record data. Costs were derived from national pay scales and literature. The model did not include costs which remain the same before and after the introduction of online self-sampling service (e.g. diagnostic costs when the service is provided in-house). When the diagnostic tests are provided externally via a commercial provider, we assumed that only $30 \%$ or $50 \%$ of the test costs (marginal costs) could be released by microbiology.

Results The average weekly staff cost for managing 227 male and 237 female clients was $£ 3104$ and $£ 3075$, respectively. $50 \%$ of 76 male and 78 female asymptomatic clients using the online self-sampling services resulted in: a weekly cost saving of $£ 261$ and $£ 49$ respectively when provided in-house, and ranged from a weekly cost saving of $£ 103$ to an additional $£ 241$ for male pathways and an additional $£ 126-£ 479$ for female pathways when provided externally.

Conclusions The introduction of online-based self-sampling for asymptomatic patients may reduce the overall cost when provided in-house but not externally.

\section{P318 FREQUENT DETECTION OF SHIGELLA IN MSM ALSO IN THE ABSENCE OF CLINICAL SYMPTOMS}

\footnotetext{
${ }^{1,2} \mathrm{~A}$ Van Dam* ${ }^{1}$ IJ Braam, ${ }^{1} \mathrm{M}$ Hoogeland, ${ }^{3,4} \mathrm{H}$ De Vries, ${ }^{1} \mathrm{~S}$ Bruisten. ${ }^{1}$ Amsterdam Health Service, Public Health Laboratory, Amsterdam, The Netherlands; ${ }^{2}$ Medical Microbiology, Amsterdam UMC, Amsterdam, The Netherlands; ${ }^{3}$ STI Clinic, Amsterdam Health Service, Amsterdam, The Netherlands; ${ }^{4}$ Dermatology, Amsterdam UMC, Amsterdam, The Netherlands
}

10.1136/sextrans-2021-sti.373

Introduction Shigellosis can present as a severe infection, including bloody diarrhea. About $22 \%$ of reported shigellosis 